\title{
Subthalamic stimulation for Parkinson disease: determination of electrode location necessary for clinical efficacy
}

\author{
Shearwood McClelland III, A.B., Blair Ford, M.D., Patrick B. Senatus, M.D., Ph.D., \\ Linda M. Winfield, R.N., M.P.H., Yunling E. Du, PH.D., Seth L. Pullman, M.D., \\ QiPIng Yu, Ph.D., Steven J. Frucht, M.D., Guy M. MCKhann II, M.D., \\ ANd Robert R. Goodman, M.D., Ph.D.
}

\begin{abstract}
Departments of Neurological Surgery and Neurology, Columbia University College of Physicians and Surgeons; and Center for Biostatistics and Epidemiology, Columbia University Mailman School of Public Health, New York, New York
\end{abstract}

\begin{abstract}
Object. Deep brain stimulation (DBS) of the subthalamic nucleus (STN) performed using intraoperative microelectrode recording (MER) to adjust electrode placement has become a widely used treatment for patients with advanced Parkinson disease (PD). Few studies have been conducted to examine the location of implanted electrodes relative to the intended target, and even fewer have been undertaken to investigate the degree to which variations in the location of these electrodes impacts their clinical efficacy. This study was performed to examine these issues.

Methods. The authors located 52 bilaterally implanted DBS electrode tips on postoperative magnetic resonance (MR) images obtained in 26 consecutive patients. Postoperative and preoperative planning MR images were merged to determine the DBS electrode tip coordinates relative to the midcommissural point. Surgical records listed the intended target coordinates for each DBS electrode tip. Clinical outcome assessment included the Unified PD Rating Scale (UPDRS) motor score at 1 year, standardized questionnaires, and routine follow-up visits.

The mean difference between electrode tip location and intended target for all 52 electrodes was less than $2 \mathrm{~mm}$ in all axes. Only one electrode was farther than $3 \mathrm{~mm}$ from the intended target, and this was the only electrode that had to be replaced due to lack of clinical efficacy (lack of tremor suppression); its reimplantation $4 \mathrm{~mm}$ more medially provided excellent tremor control. High correlation coefficients indicate that the MR imaging analysis accurately determined the anatomical location of the electrode tips. Blinded videotape reviews of UPDRS motor scores comparing effects of stimulation in patients who were "on" and "off" medication identified subgroups in whom there was minimal and maximal stimulation response. Patients in these subgroups had no differences between the MR imaging-determined actual electrode tip location and its intended location. Similarly, improvements of dyskinesias and severity of symptoms encountered during the wearing-off period for the drug did not correlate with variations of electrode tip location.

Conclusions. The findings in this study lead the authors to suggest that a DBS electrode placed anywhere within a 6-mm-diameter cylinder centered at the presumed middle of the STN (based on stereotactic atlas coordinates) provides similar clinical efficacy. Future studies may be warranted to evaluate prospectively the degree to which MER modification of the anatomically and/or image-determined target improves clinical efficacy of DBS electrodes.
\end{abstract}

\section{KeY WoRdS • deep brain stimulation • Parkinson disease • subthalamic nucleus • magnetic resonance imaging $\bullet$ outcome}

Parkinson disease is characterized by the loss of dopaminergic cells in the substantia nigra pars compacta. ${ }^{10} \mathrm{Al}-$ though levodopa therapy generally provides an excellent clinical response for years, many patients eventually experience progressive worsening, disabling motor fluctuations, and dose-limiting dopaminergic dyskinesias. ${ }^{5,17}$ For medically refractory $\mathrm{PD}$, continuous electrical stimulation of the

Abbreviations used in this paper: $\mathrm{AC}-\mathrm{PC}=$ anterior commissure-posterior commissure; CRW = Cosman-Roberts-Wells; DBS = deep brain stimulation; MER = microelectrode recording; $\mathrm{MR}=$ magnetic resonance; $\mathrm{PD}=$ Parkinson disease; $\mathrm{SD}=$ standard deviation; $\mathrm{SNR}=$ substantia nigra pars reticulata; $\mathrm{STN}=$ subthalamic nucleus; UPDRS = Unified PD Rating Scale; 3D = three-dimensional.
STN can lead to sustained improvements in tremor, bradykinesia, rigidity, dyskinesias, motor fluctuations as the medications wear off, and reduction in medication requirements. ${ }^{6,11-13,15,16}$

The outcome of DBS is believed to be critically dependent on accurate targeting of the STN. ${ }^{19,24}$ To date, the best method of target localization and electrode implantation remains uncertain. In many centers, including ours, surgeons routinely use electrophysiological mapping with singleunit MERs to locate, measure, and map the STN and to compare it with standardized atlas sections obtained in the sagittal and coronal planes., ${ }^{4,23,25}$ The accuracy of electrode placement has been studied, ${ }^{23,29}$ but the precise relationship 
between targeting accuracy and clinical outcome remains unclear. , $19,28^{2}$

In this study we measured the accuracy of electrode targeting in three dimensions, based on postoperative MR imaging. We used an estimate of electrode placement accuracy derived from the imaging data to determine the relationship between the accuracy of electrode placement and the clinical outcome of STN stimulation.

\section{CLINICAL MATERIAL AND METHODS}

\section{Patient Selection}

Between January 2000 and April 2001, 26 consecutive patients (17 men and nine women) with advanced PD underwent bilateral STN DBS electrode placement at our institution. Twenty-five patients underwent bilateral simultaneous implantation, and one had a staged procedure. Patients were selected based on their history of longstanding levodopa-responsive PD syndrome with motor fluctuations consisting of periods of severe immobility ("offs"), periods with good motor function ("good ons"), and levodopa-induced dyskinesias that occurred despite optimal medication adjustment, as detailed in reports published elsewhere. ${ }^{3,6,8,24}$ Neuropsychological testing ruled out significant cognitive impairment. All patients underwent extensive preoperative evaluation, including detailed neurological examinations and volumetric MR images of the brain. The mean age of the patients at the time of surgery was 59.3 years (range 31-79 years), and the mean approximate duration of PD was 14 years (range 4-25 years). Of our 26 patients, six had undergone a previous operation for PD (four pallidotomies and three fetal tissue transplants; one patient had undergone both operations).

\section{Stereotactic Planning}

A functional CRW stereotactic frame (Radionics, Inc., Burlington, MA) was used for all 26 patients. Efforts were made to align the stereotactic ring with the orbitomeatal plane. A volumetric $\mathrm{T}_{1}$-weighted $\mathrm{MR}$ image was obtained in the axial plane, which included the region of the AC-PC plane, with 1.5- or 2-mm slice thickness. For the first nine patients, StereoPlan software (Radionics, Inc.) was used for surgical planning. The initial target was calculated relative to the intercommissural midpoint ( $4 \mathrm{~mm}$ posterior, $4 \mathrm{~mm}$ inferior, and $12 \mathrm{~mm}$ lateral), adjusting for tilt in all three planes. For the subsequent 17 patients, Stealth FrameLink 2.0 software (Medtronic, Inc., Minneapolis, MN) was used. The FrameLink 2.0 program reformatted the MR image to the AC-PC plane to yield the calculated coordinates for the STN.

\section{Neurophysiological Localization}

High-impedance (range 200-700 kOhm) 24-mm-long tungsten microelectrodes with a tip size of 20 to $25 \mu \mathrm{m}$ (FHC, Inc., Bowdoinham, ME) were advanced to the end of the guide tube, which was located $30 \mathrm{~mm}$ above the calculated anatomical target. Impedance at $1000 \mathrm{~Hz}$ was measured at 3 and $20 \mathrm{~mm}$ after the microelectrode exited the cannula. Subsequently, the recording microelectrode was advanced to the target by an electronic stepper microdrive. The electrical signals from single neuronal units and back- ground activity were then filtered at 100 to $3000 \mathrm{~Hz}$, preamplified, amplified 10,000 to 50,000 times, digitized, sent to an oscilloscope and audio system for real-time monitoring and then to a digital recording device for offline analysis. The MER coordinate positions, Fourier transforms of discharge frequencies, and the action potential morphologies of single units, fiber activity, and background changes were quantified and recorded. Typically, bursting cells of the anterior thalamus were first encountered, followed by electrically quieter regions corresponding to the fields of Forel and zona incerta. Proceeding ventrally, an area of increased background noise and irregularly firing neurons (often responsive to movement) were detected; these corresponded to the STN. Farther ventrally, below the STN, a region of more rapidly and regularly firing neurons corresponding to the SNR was detected. The mean STN height along the first microelectrode track was $4.9 \pm 0.8 \mathrm{~mm}$ (range 2.5-6.4 mm).

The height and depth of the STN and the depth of the SNR were compared with those expected for a track through the target point on the stereotactic atlas along the known angle of the trajectory. ${ }^{20}$ If these data indicated that the trajectory was as expected in the central region of the STN (this included a minimum 4-mm length of STN), then these results would be used for DBS electrode placement. The first MER track satisfied these criteria on the first side in 13 of 26 implants. In these patients, the DBS electrode was placed $1 \mathrm{~mm}$ anterior to the calculated target path. In the remaining 13 first-side implants, one or more parallel MER tracks were made until the appropriate criteria for the central region of the STN had been adequately identified. On the second side, 24 of 26 implants were done with a single MER track. Typically, this track was a mirror image to that used for the DBS electrode on the first side. The final placement for 40 electrodes $(77 \%)$ was adjusted either in the $\mathrm{x}$ (lateral/medial) or $\mathrm{y}$ (anterior/posterior) plane relative to the initial stereotactic target (Table 1). These $\mathrm{x}$ and $\mathrm{y}$ adjustments were made with the FHC microdrive to create a trajectory parallel to the initial one. The electrode was placed to a final depth (z plane) such that the bottom of the deepest contact was just above the top of the SNR. The final depth beyond the calculated stereotactic target varied from 1 to $4 \mathrm{~mm}$, with a mean depth of $2.5 \pm 0.62 \mathrm{~mm}$ (mean \pm $\mathrm{SD}$ ). The $\mathrm{x}$ and $\mathrm{y}$ adjustments and the depth for each DBS electrode were used to calculate the intended tip location.

After MER, the guide sleeve was removed, the stereotactic apparatus was set for the desired trajectory, a longer

TABLE 1

Operating room adjustments to coordinates to determine intended DBS electrode tip location in 26 patients with $P D^{*}$

\begin{tabular}{cc}
\hline \hline Adjustment of $\mathrm{x}, \mathrm{y}$ Axes $(\mathrm{mm})$ & No. of Electrodes w/ Adjustment \\
\hline $0,1.5 \mathrm{pst}$ & 1 \\
0,0 & 12 \\
0,1 ant & 28 \\
$0,1.5$ ant & 4 \\
0,2 ant & 3 \\
1 lat, 0 & 2 \\
0.5 lat, 0 & 1 \\
1.5 lat, 1 ant & 1 \\
total & 52 \\
\hline
\end{tabular}

$*$ Ant $=$ anterior; pst $=$ posterior. 
guide sleeve was inserted (15 $\mathrm{mm}$ above the target), and the DBS electrode (model 3387 or 3389; Medtronic, Inc.) was then placed. Macrostimulation (3 V, pulse width $60 \mu \mathrm{sec}$, $185 \mathrm{~Hz}$, bipolar configuration between the deepest and most superficial electrodes) produced no sustained sensory symptoms or dystonic muscle contractions in any patient. Individuals with significant bradykinesia and rigidity at the time of macrostimulation testing had an observable improvement when assessed using these stimulation parameters. Electrode placement was never altered based on macrostimulation findings.

\section{Stimulator Settings for STN}

Typically, pulse generators were implanted 1 to 2 weeks after the DBS electrodes were placed, with initial programming occurring 1 week later. Subsequent extended programming sessions were typically conducted at the 1- and 3-month follow-up visits, with the patient in an "off" (unmedicated) state. The final settings were those that produced the greatest improvement in tremor (if present), bradykinesia, and rigidity, without adverse or intolerable side effects. Stimulator setting data were obtained from chart review. Medications were regularly adjusted to optimize control of PD symptoms.

\section{Merging of Pre-and Postoperative MR Images}

The postoperative and immediate preoperative MR images for each of the 26 patients were transferred to DAT tapes and then to the Stealth workstation (Medtronic, Inc.). The Stealth FrameLink 4.0 program was used to manually merge the frame-based (preoperative) and postoperative volumetric MR images (eight to 10 anatomical landmarks were used). The accuracy of the merge was confirmed using the Split format; this format allows the user to examine carefully two overlapping images by sweeping across the screen to convert from one to the other.

The mean point-to-point matching error was less than 1 $\mathrm{mm}$, with an excellent overlap in all three planes of view when using the Split format. The mean error of merging for all 26 patients was $0.39 \mathrm{~mm}$ (range $0.12-0.83 \mathrm{~mm}$ ). After the merge, we set the Blend setting to $0 \%$ on the merged image, which displayed only the preoperative image with its fiducial markers (not the DBS artifact seen on the postoperative views). We then obtained the coordinates for the CRW fiducial frame, and found that the mean fiducial error for all 26 patients was $0.73 \mathrm{~mm}$ (range $0.22-0.97 \mathrm{~mm}$ ).

\section{Manual Calculation of STN Target Compared With FrameLink Guidance}

The MR image was then reformatted to the AC-PC plane by identifying these two commissures and three midline landmarks (to correct for tilt). The AC-PC distance was recorded for each patient, then we shifted the Blend setting from 0 to $100 \%$, to visualize the postoperative MR images only. All planes of view were used to determine the center at the tip of the electrode MR image artifact. In addition to the stereotactic frame coordinates of the point itself, its coordinates relative to the intercommissural midpoint were recorded (termed AC-PC coordinates). Therefore, there were four sets of coordinates for each patient, which consisted of the DBS electrode tip stereotactic frame coordinates and the
AC-PC coordinates for left and right. We then obtained the STN target coordinates (stereotactic frame and AC-PC coordinates) for the left and right side of each patient; these were calculated using FrameLink 4.0. This procedure for determining the DBS electrode tip coordinates was performed by three separate observers (S.M., P.B.S., and R.R.G.) to test the interobserver reliability of the definition of AC-PC distance, the manual coordinates, and the computer coordinates (which relied in part on each observer's definition of the AC-PC distance).

\section{Calculation of STN Coordinates for Intended DBS Target}

We used the CRW arc and ring angles relative to the AC-PC plane, the $\mathrm{x}$ and $\mathrm{y}$ axis shifts from the CRW target, and the depth of the electrode placement to calculate the intended DBS electrode tip target for each patient, which we defined as the ventral-most portion of the electrode artifact visible on MR images. The arc angles for the 26 patients ranged from 9 to $22^{\circ}$, with a mean of $16.8^{\circ}$. The forward ring angle relative to the $\mathrm{AC}-\mathrm{PC}$ was $30^{\circ}$ for each electrode.

We then used the FrameLink 4.0 workstation with the operating room adjustments (that is, the $\mathrm{x}$ - and $\mathrm{y}$-axis shifts and the depth of placement) to determine the intended target for the electrode tip in relation to the midpoint of the patient's AC-PC line. We adjusted the initial y coordinate (which is 4 $\mathrm{mm}$ posterior to the midcommissural point) by a factor of cosine $30^{\circ}$, which equals 0.866 (we rounded to 0.9 ). For example, if a patient had an operating room adjustment of $1.5 \mathrm{~mm}$ anterior in the $\mathrm{y}$ axis, this value was adjusted by $1.5 / 0.9=1.7$. If the operating room adjustment was anterior, then the value was added to the initial y coordinate, and if it was negative it was subtracted from that coordinate. Therefore, in this example, if the operating room adjustment was anterior, the FrameLink 4.0 adjustment in the y axis for the AC-PC coordinate would be $-4.0+1.7=-2.3$. If the operating room adjustment was posterior, then the FrameLink 4.0 adjustment would be $-4.0-1.7=-5.7$. After these adjustments, we were then able to determine the corresponding $\mathrm{AC}-\mathrm{PC}$ coordinates for the desired STN, which was determined in the operating room for each patient. A similar adjustment was made for the $\mathrm{x}$ axis by using the arch angle relative to the $\mathrm{AC}-\mathrm{PC}$ plane.

\section{Difference Between Intended and MR Imaging-Confirmed DBS Electrode Tip Target}

To determine the difference between the intended and the measured DBS electrode tip target, we compared the AC-PC tip coordinates of each by subtracting the intended AC-PC coordinates from the MR imaging-confirmed AC-PC coordinates in each plane ( $\mathrm{x}, \mathrm{y}$, and $\mathrm{z}$ ) for all 26 patients. We then calculated the distance between the tip coordinates in 3D space for each patient by taking the square root of $\left(x^{2}+y^{2}+z^{2}\right)$. For example, one patient's right electrode was $-0.4 \mathrm{~mm}$ in the $\mathrm{x},-2.2 \mathrm{~mm}$ in the $\mathrm{y}$, and $+1.8 \mathrm{~mm}$ in the $\mathrm{z}$ plane from the intended target. Therefore, her Right 3D coordinate was the square root of $\left([-0.4]^{2}+[-2.2]^{2}+[+1.8]^{2}\right)=2.9 \mathrm{~mm}$. As a result, there were eight values for each patient, as follows: Right x, Right y, Right z, Right 3D coordinate, Left x, Left y, Left $z$, and Left 3D coordinate. These values were obtained for each of the three independent observers (to de- 
S. McClelland III, et al.

termine the interobserver reliability) and repeated by one observer (R.R.G.) in a subset of patients (to determine intraobserver reliability).

\section{Measures of Clinical Outcome}

Postoperative evaluation consisted of a neurological examination in which the Core Assessment Program for Intracerebral Transplantations ${ }^{14}$ protocol, postoperative MR imaging, and assessment of PD symptoms and medications ${ }^{9}$ were used. Clinical outcome at more than 5 months postoperatively was measured using a novel self-assessment questionnaire (Appendix) administered either through the mail and/or by telephone interview. Symptoms assessed included falling, freezing, tremor, the presence of significant dyskinesias, and sensory complaints. Activities assessed as a subset of the UPDRS ${ }^{7}$ included speech, salivation, swallowing, handwriting, cutting food, dressing, bathing, turning in bed, walking, and overall activities of daily living. ${ }^{21}$ Information sought included the laterality of symptoms, duration and quality of "on" periods, and the presence, severity, and duration of "off" periods and dyskinesias. Patients were asked to compare these responses to their preoperative condition. At 1 year postoperatively, 21 of the 26 patients underwent a videotaped examination to allow a "blinded" review of clinical signs (UPDRS III [motor subscale]). This blinded evaluation of the change in UPDRS motor score between pre- and postoperative assessments was performed as part of an independent study of our patients who received STN implants (which happened to include 21 of the 26 patients involved in this study), and has been reported separately. ${ }^{8}$ Further clinical results were obtained using a standard questionnaire (supplemented by telephone interview) and through routine clinical follow-up visits and chart review.

\section{Statistical Analysis}

Statistical analysis was performed using Statistical Product and Service Solutions version 10.0 software (SPSS, Inc., Chicago, IL). Each series of comparisons between outcome measures was conducted using paired two-sample t-tests for approximately normally distributed data, and nonparametric Wilcoxon signed-rank tests for discrete outcomes. Nonparametric measures of correlation were performed using the Kendall tau and Spearman rho tests. To identify outcome predictors, regression analyses were performed.

\section{RESULTS}

\section{Electrode Tip Locations}

Difference Between Actual Location and Intended Target. The mean difference between electrode tip location and intended target for all 26 patients was less than $2 \mathrm{~mm}$ in all axes regardless of laterality, with a relatively small range of tip locations (Table 2). The mean absolute deviation of electrode tip location from target was $1.4 \mathrm{~mm}$ in the $\mathrm{x}$ axis, $1.2 \mathrm{~mm}$ in the $\mathrm{y}$ axis and $1.9 \mathrm{~mm}$ in the $\mathrm{z}$ axis. In the $\mathrm{x}$ axis, 41 of the 52 electrodes ( 21 right, 20 left) were located no farther than $2 \mathrm{~mm}$ from the intended target, 43 of 52 (22 right, 21 left) in the y axis, and 30 of 52 (15 right, 15 left) in the $\mathrm{z}$ axis. Only one electrode (left side) was located far-
TABLE 2

Location of electrode tips relative to intended target $(\mathrm{mm})$ in 26 patients with $P D^{*}$

\begin{tabular}{|c|c|c|c|c|}
\hline \multirow{3}{*}{$\begin{array}{l}\text { Side/ } \\
\text { Axis }\end{array}$} & \multicolumn{4}{|c|}{ Tip Location (52 sides) } \\
\hline & \multicolumn{2}{|c|}{ Mean $\pm \mathrm{SD}$} & \multicolumn{2}{|c|}{ Range } \\
\hline & $\mathrm{Rt}$ & $\mathrm{Lt}$ & Rt & $\mathrm{Lt}$ \\
\hline $\mathrm{X}$ & $1.3 \mathrm{med} \pm 1.1$ & $1.3 \mathrm{med} \pm 1.2$ & 3.0 med-2.5 lat & 0.4 lat $-5.1 \mathrm{med}$ \\
\hline $\mathrm{y}$ & 0.5 pst \pm 1.4 & $0.8 \mathrm{pst} \pm 1.5$ & 3.4 pst-2.0 ant & $4.1 \mathrm{pst}-1.2 \mathrm{ant}$ \\
\hline $\mathrm{Z}$ & $1.7 \sup \pm 1.6$ & 1.6 sup \pm 2.0 & $1.2 \mathrm{inf}-6.6 \mathrm{sup}$ & 3.8 inf-5.2 sup \\
\hline $3 \mathrm{D}$ & $2.9 \pm 1.4$ & $3.2 \pm 1.5$ & $0.7-6.7$ & $1.0-5.9$ \\
\hline
\end{tabular}

$*$ Inf = inferior; med = medial; sup = superior.

ther than $3 \mathrm{~mm}$ from the intended target in the $\mathrm{x}$ axis, as were four of 52 electrodes in the y axis (two right, two left) and 12 of 52 in the $\mathrm{z}$ axis (four right, eight left). From a 3D vantage point, only one of the 26 electrodes on the right side was placed more than two SDs from the mean of 2.9 $\mathrm{mm}$, whereas none of the 26 electrodes on the left side was placed more than two SDs from the mean of $3.2 \mathrm{~mm}$. A graphic depiction of the DBS electrode tip locations on the right side (Fig. 1) demonstrates the clustering near the intended target, with only three of the 26 electrode tips identified outside the rectangular volume encompassing two SDs from the mean of intended target. This was also true for the DBS electrodes on the left side (not shown).

Measurement of Inter- and Intraobserver Correlation and Reliability. The interobserver reliability of the electrode tip coordinates among the three observers was assessed. Reliability was high in the $x$ and $y$ axes $(\geq 0.85)$, slightly lower in the $\mathrm{z}$ axis $(\geq 0.7)$, and statistically significant for the Right x, Left x, Left y, Right 3D, and Left 3D coordinates. In the Right y, Right $\mathrm{z}$, and Left $\mathrm{z}$ axes, however, interobserver reliability was significantly different $(p<0.05)$. In each of these cases, two of the three observers agreed, with the third observer's discrepancy in measurement (Right $\mathrm{y}=$ $0.3 \mathrm{~mm}$; Right and Left $\mathrm{z}=1.1 \mathrm{~mm}$ ) resulting in the statistically significant difference. The degree of correlation between the three observers was extremely high for every coordinate, because the interclass correlation coefficient was never less than 0.9 , regardless of laterality.

Intraobserver reliability (in all axes and 3D distances) was calculated using the coordinates of one observer (R.R.G.). No intraclass correlation coefficient was less than 0.8 , with the exception of the Right $\mathrm{z}$ axis (0.35).

Comparison of Accuracy Between First and Second Sides Implanted. Of our 26 patients who underwent bilateral implantation, in 19 the right electrode was implanted first, and in the other seven it was implanted second. We compared the electrode locations between these two groups of patients to determine the existence of a significant discrepancy in electrode position between them. Statistical analysis revealed no significant difference between the two groups in any axis.

We also compared the electrode placement between the first and second sides implanted for all 26 patients (taking into account the differences in lateral-medial axis measurement between sides). Statistical analysis revealed no significant difference between the two sides for electrodes implanted in any axis. 


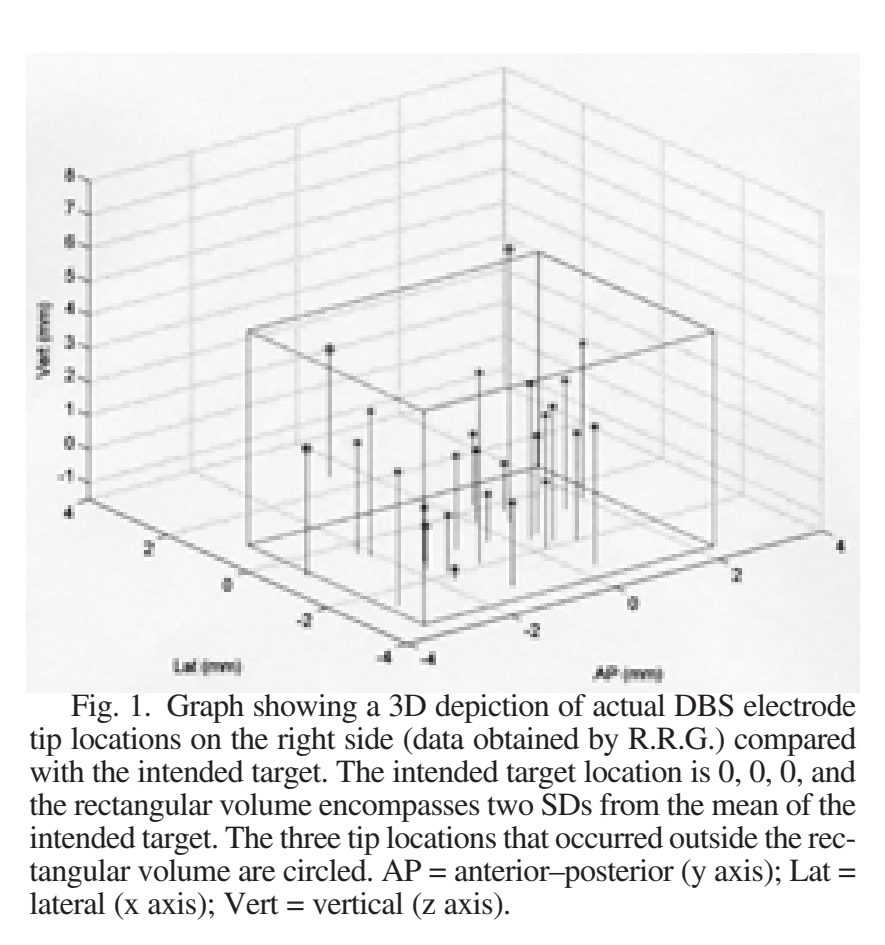

Comparison of Accuracy in Relation to Number of Microelectrode Passes. To determine if electrode placement accuracy was correlated with the number of passes, we also compared patients receiving a single MER pass with those who received multiple passes (Table 3). On the right side, 15 patients underwent implantation after a single MER pass, whereas the other 11 received implants after multiple passes. The two-tailed t-test for equality of means revealed a significant difference between the groups only in the Right $\mathrm{x}$ axis $(\mathrm{p}=0.035)$, with the patients who underwent multiple passes receiving their implants $0.93 \mathrm{~mm}$ closer to the intended target. On the left side, 22 patients had a single MER pass, whereas the other four had multiple passes (two had two passes and the other two had three passes). Statistical analysis revealed a significant difference between the groups only in the Left $\mathrm{z}$ axis $(\mathrm{p}=0.04)$.

In the order of side implanted, 13 of the 26 patients had multiple MER passes on the first side. Of the second sides implanted, only two of the 26 patients had multiple passes (one of whom was the patient who underwent implants on separate days). Of the 26 second sides implanted, in 18 an intraoperative adjustment was made in the $\mathrm{x}$ and $\mathrm{y}$ axes that was identical to that made on the first side implanted. Six of the eight asymmetrically adjusted second sides were adjusted $1 \mathrm{~mm}$ in the $\mathrm{x}$ and/or $\mathrm{y}$ axis. One second side was adjusted $1.5 \mathrm{~mm}$ in the $\mathrm{x}$ axis, and the other was adjusted 2.5 $\mathrm{mm}$ in the $\mathrm{y}$ axis.

Comparison of Accuracy Between StereoPlan and FrameLink 2.0-Guided Implants. During the 15 months over which our 26 patients received implants, we changed our guidance system from StereoPlan to FrameLink 2.0. Of the 26 patients, the first nine underwent implantation aided by StereoPlan and the last 17 with FrameLink 2.0. We compared the electrode placement in all axes between the two guidance systems, to rule out a systematic difference in targeting. Statistical analysis revealed a significant difference between the two systems in the Right $x$ axis $(p=0.031)$, Right $\mathrm{z}$ axis $(\mathrm{p}=0.001)$, and Right 3D coordinates $(\mathrm{p}=0.015)$. Of these three axes, the Right $x$ was the only one in which the StereoPlan was closer to the intended target (1 mm closer). In the Right $\mathrm{z}$ the FrameLink 2.0 was $2 \mathrm{~mm}$ closer to the intended target, and in the Right 3D the FrameLink 2.0 was $1.3 \mathrm{~mm}$ closer to the intended target. No significant difference was found between the two systems in the Right y, Left x, Left y, Left z, and Left 3D axes.

\section{Clinical Outcome}

Electrode Contacts Used for Stimulation. Stimulator settings were available for all 52 electrodes (range 1-14 months postoperatively). Table 4 indicates the active electrode contacts. The mean distance between the active electrode contact and the electrode tip was $5.5 \mathrm{~mm}$, placing the average location of stimulation between the central location of Contacts 1 and 2. This region of the electrode (Contact 1 and/or 2) was active in 48 of the 52 electrodes, consistent with previous reports. ${ }^{8,26}$ Of the 10 electrodes programmed in a bipolar mode (with case off), only two used the number 3 contact as a negative/active contact (both electrodes were in the same patient). The coordinates for these two electrode tips were analyzed and found to be within one SD of the mean in the $\mathrm{x}$ or $\mathrm{z}$ axes bilaterally (Right $\mathrm{z}=3.1 \mathrm{~mm}$, mean $1.7 \pm 1.6 \mathrm{~mm}$; Left $\mathrm{z}=1.1 \mathrm{~mm}$, mean $1.6 \pm 2 \mathrm{~mm}$ ). For the right electrode, the y coordinate was more than one $\mathrm{SD}$ anterior to the mean (Right $\mathrm{y}=2 \mathrm{~mm}$, mean $-0.5 \pm$ $1.4 \mathrm{~mm}$ ), whereas the Left y coordinate was within one SD of the mean (Left y $=0.2 \mathrm{~mm}$, mean $-0.8 \pm 1.5 \mathrm{~mm}$ ).

Comparison of Most- and Least-Improved Patients on Follow-Up Videotape. Videotape reviews were performed at 1 year postoperatively in 21 of the 26 patients, and clinical outcomes were graded by a movement disorder neurologist (S.J.F.) who was blinded to the electrode tip location. Videotaped analysis is a highly objective evaluation of clinical outcome, and has been relied on in other studies as a way to determine the clinical efficacy of stimulation. ${ }^{18,22}$

In the patients with videotaped follow-up visits, the percentage change in UPDRS III motor scores (assessed based on blinded videotape review) was determined by comparing the on-stimulation, off-medication state with the off-stimulation, off-medication state at 1 year. An improvement of less than $10 \%$ was characterized as limited improvement,

\section{TABLE 3}

Location of electrode tips relative to intended target ( $\mathrm{mm}$ ), comparing single with multiple microelectrode tracks

\begin{tabular}{|c|c|c|c|c|}
\hline \multirow{3}{*}{$\begin{array}{l}\text { Side/ } \\
\text { Axis }\end{array}$} & \multicolumn{4}{|c|}{ Tracks $($ mean $\pm \mathrm{SD}) *$} \\
\hline & Rt & $\mathrm{Lt}$ & Rt & $\mathrm{Lt}$ \\
\hline & Single & Multiple & Single & Multiple \\
\hline $\mathrm{x}$ & $1.6 \mathrm{med} \pm 0.8$ & $0.7 \mathrm{med} \pm 1.3$ & $1.4 \mathrm{med} \pm 1.2$ & 0.9 med \pm 0.7 \\
\hline $\mathrm{y}$ & $0.6 \mathrm{pst} \pm 1.2$ & 0.3 pst \pm 1.7 & $0.8 \mathrm{pst} \pm 1.5$ & 0.7 pst \pm 1.4 \\
\hline $\mathrm{z}$ & 1.5 sup \pm 1.8 & $2.0 \mathrm{sup} \pm 1.4$ & $1.4 \sup \pm 2.1$ & $2.8 \sup \pm 0.8$ \\
\hline $3 \mathrm{D}$ & $2.8 \pm 1.6$ & $3.0 \pm 1.1$ & $3.1 \pm 1.6$ & $3.3 \pm 1.0$ \\
\hline
\end{tabular}

* On the right side, which was attempted first, 15 electrodes were implanted in a single pass and 11 were placed after multiple tries. On the left side, 22 electrodes were implanted in a single pass and four were placed after multiple attempts. 
TABLE 4

Distribution of active programming electrode contacts used for stimulation

\begin{tabular}{lr}
\hline \multicolumn{1}{c}{ Active Electrode(s) } & No. \\
\hline monopolar (case positive) & 4 \\
0 only & 16 \\
1 only & 6 \\
2 only & 0 \\
3 only & 4 \\
0 \& 1 only & 1 \\
0 \& 2 only & 0 \\
0 \& 3 only & 6 \\
1 \& 2 only & 0 \\
1 \& 3 only & 3 \\
2 \& 3 only & 2 \\
1 2, \& 3 & 42 \\
total & \\
bipolar (case off) & 3 \\
1 positive, 2 negative & 2 \\
1 negative, 2 positive & 2 \\
2 positive, 3 negative & 1 \\
0 positive, 1 negative, 2 positive & 1 \\
0 negative, 1 positive, 2 negative & 1 \\
1 positive, 2 negative, 3 positive & 10 \\
total &
\end{tabular}

whereas improvement greater than $40 \%$ was classified as maximal improvement. Of the 21 patients who were analyzed with the aid of videotapes, four were classified as having limited improvement, whereas five were deemed maximally improved. The difference between electrode tip location and intended target (for each of the three blinded observers) was compared between these groups to evaluate the existence of a consistent trend separating one group from the other (Table 5 contains the data collected by one observer, R.R.G.). Although there were no statistically significant differences in the axes, it was noteworthy that in five of the eight axes, the patients with limited improvement had a greater discrepancy between the electrode tip and the intended target than the ones who attained maximal improvement. This finding was highly correlated among the three observers.

Questionnaire Results. From the questionnaire data, we were able to determine several aspects of patient symptomatology postoperatively compared with preoperatively. The first aspect was patient self-assessment of surgical effect on laterality of PD symptoms. Eighteen of the 26 patients reported experiencing preoperative symptoms that were greater on one side, and of these 18, 10 had symptoms whose laterality was not altered by electrode placement, seven had symptoms whose laterality was abolished by electrode placement, and one had symptoms whose laterality reversed following electrode placement. The mean electrode tip location in the 10 unaltered patients was compared with the tip location in the patient whose laterality reversed, to determine if the reversal could be explained by electrode placement. Both the right and left electrodes were within two SDs of the mean of the other 10 patients in all axes. In addition, there was no trend noted for a difference in agreement between the right and left electrodes.

A second aspect was patient self-assessment of dyskinesias. Twenty-four of the 26 patients reported experiencing severe dyskinesias preoperatively. Postoperatively, 15 of these 24 reported significant improvement in dyskinesias in comparison with preoperative status, six reported moderate improvement, and three reported no improvement in dyskinesias. The mean of the electrode locations in the 15 who experienced significant improvement was compared with the locations in the three who did not improve to elucidate the role of electrode placement in producing these clinical outcomes. Of the three patients who did not improve, one had electrode placement well outside two SDs of the mean for the Right 3D electrode, due to a Right $\mathrm{z}$ electrode placed nearly four SDs from the mean. In all three patients, the $\mathrm{X}$ and $y$-axis differences were less than $2 \mathrm{~mm}$ from the mean.

A third aspect elicited was patient self-assessment of the severity of off-medication symptoms. All 26 patients reported experiencing severe off-medication symptoms preoperatively. Postoperatively, 17 reported significant decreases in off-medication symptom severity, seven reported no decrease, and two reported a moderate decrease. The mean of the electrode locations in the 17 patients whose condition significantly improved was compared with the mean of the locations in the seven whose condition did not to elucidate the role of electrode placement on these clinical outcomes. Of the seven patients who did not improve, only one had an axis where electrode placement was more than two SDs from the mean recorded in the 17 patients who improved. This was in the Right $\mathrm{z}$ and Right 3D axis. This patient was the same one whose dyskinesias did not improve postoperatively (see previous paragraph).

Clinical Efficacy After Electrode Reimplantation. Of these 26 patients, one who had bilateral tremor-dominant PD experienced no improvement in his left-sided tremor. On postoperative evaluation, it was found that his right electrode placement was very lateral. His electrode was found to be the farthest outlier in the lateral (x) axis of all 26 patients. Subsequent reimplantation of a new electrode $4 \mathrm{~mm}$ medial to the prior electrode tip location target resulted in excellent tremor control.

TABLE 5

Evaluation of electrode tip location compared with intended target related to improvement in UPDRS score

\begin{tabular}{|c|c|c|c|c|}
\hline $\begin{array}{c}\text { Absolute } \\
\text { Value of } \\
\text { Tip-Target } \\
\text { Differential }\end{array}$ & $\begin{array}{c}\text { Improvement } \\
\text { on UPDRS } \\
\text { Blinded } \\
\text { Review* }\end{array}$ & No. & $\begin{array}{c}\text { Mean } \pm \mathrm{SD} \\
(\mathrm{mm})\end{array}$ & $\mathrm{p}$ Value \\
\hline \multirow[t]{2}{*}{ Rt $x$} & limited & 4 & $1.6 \pm 1.1$ & \multirow[t]{2}{*}{0.356} \\
\hline & maximal & 5 & $1.1 \pm 0.5$ & \\
\hline \multirow[t]{2}{*}{ Lt $x$} & limited & 4 & $1.1 \pm 1.0$ & \multirow[t]{2}{*}{0.728} \\
\hline & maximal & 5 & $1.3 \pm 1.1$ & \\
\hline \multirow[t]{2}{*}{ Rt y } & limited & 4 & $1.6 \pm 1.3$ & \multirow[t]{2}{*}{0.348} \\
\hline & maximal & 5 & $0.9 \pm 0.8$ & \\
\hline \multirow[t]{2}{*}{ Lt y } & limited & 4 & $1.4 \pm 1.8$ & \multirow[t]{2}{*}{0.185} \\
\hline & maximal & 5 & $0.2 \pm 0.1$ & \\
\hline \multirow[t]{2}{*}{ Rt z } & limited & 4 & $2.9 \pm 0.9$ & \multirow[t]{2}{*}{0.776} \\
\hline & maximal & 5 & $2.5 \pm 2.5$ & \\
\hline \multirow[t]{2}{*}{ Lt z } & limited & 4 & $1.4 \pm 1.3$ & \multirow[t]{2}{*}{0.167} \\
\hline & maximal & 5 & $2.4 \pm 0.8$ & \\
\hline \multirow[t]{2}{*}{ Rt 3D } & limited & 4 & $3.9 \pm 0.8$ & \multirow[t]{2}{*}{0.517} \\
\hline & maximal & 5 & $3.2 \pm 2.1$ & \\
\hline \multirow[t]{2}{*}{ Lt 3D } & limited & 4 & $2.7 \pm 1.6$ & \multirow[t]{2}{*}{0.858} \\
\hline & maximal & 5 & $2.9 \pm 1.0$ & \\
\hline
\end{tabular}

$*$ Limited $=$ less than $10 \%$ improvement; maximal $=$ greater than $40 \%$ improvement. 


\section{DISCUSSION}

The goal of STN stimulation is to inactivate the entire nucleus, and it is widely assumed that a successful outcome is aided by accurate placement of the electrode tip directly through the center of the STN. Single-cell electrophysiological recordings can chart the location and dimensions of the STN, but the recording electrode must be withdrawn from the brain and a stimulating electrode must be implanted. The final placement of the DBS electrode, therefore, is not necessarily the same location as that determined by the recording microelectrode.

The goals of this study were to test the hypothesis that DBS electrode tip location relative to an intended STN target could be accurately determined on postoperative MR imaging, and that accurate targeting would correlate with clinical benefit. The method of DBS implantation in this study was based on indirect MR targeting (AC-PC stereotactic coordinates) supplemented by MER, similar to that performed at several other centers. ${ }^{3,13,24,30}$ As previously described, ${ }^{24}$ we defined the center of the MR imaging artifact (using three planes of view) as the tip of the DBS electrode.

We were able to determine with precision the stereotactic and anatomical location of 52 electrodes implanted bilaterally in 26 consecutive patients who underwent DBS of the STN. The mean distance from the intended target was no more than $2 \mathrm{~mm}$ in any dimension, a finding similar to that previously reported. ${ }^{19,24,27,30}$ Very few DBS electrode tips were found to rest more than $3 \mathrm{~mm}$ from the intended target in any single axis. In the $\mathrm{x}$ and $\mathrm{z}$ axes, we found a systematic discrepancy between the measured and intended tip location. The measured tip location shifted a mean of $1.3 \mathrm{~mm}$ medially and 1.6 to $1.7 \mathrm{~mm}$ ventrally, suggesting a shift in apparent tip location due to a DBS-produced MR imaging artifact, or perhaps a systematic error in the calculation of the intended tip location.

The intended target referred to in this study was adjusted from the image-calculated target (based on MER results) by $1 \mathrm{~mm}$ anterior (3 $\mathrm{mm}$ posterior to the intercommissural midpoint) in the majority of cases ( 28 of 52 electrodes, see Table 1). Interestingly, 22 of the remaining 24 electrodes were adjusted no more than $1 \mathrm{~mm}$ away from this target. It seems likely that these adjustments were too small to alter the clinical efficacy of these electrodes. This hypothesis is supported by our finding that there was no clear difference in clinical efficacy associated with the observed deviation of the DBS electrode tip location from the intended target. In addition, as noted in the Results section, the relationship of the active contact to the electrode tip was relatively constant; the active region of the electrode was the same in 48 $(92.3 \%)$ of 52 electrodes. It is important to note that the mean distance of this variation was of greater magnitude than the intentional adjustment. The majority of electrodes (46 of 52) fell within two SDs of the mean of the intended target (see Fig. 1 for the electrodes on the right side). Of the six electrodes that fell outside of this range, only one was found to lack clinical efficacy and require revision. This observation lends further support to the possibility that the small adjustments made based on MER do not significantly impact clinical efficacy.

In our study, the DBS electrode tips were all tightly clustered within a short distance from the intended targets, averaging no more than $1.7 \mathrm{~mm}$ in any single axis (see also
Table 2). This degree of accuracy is similar to that in a previously published report, ${ }^{24}$ in which differences between predicted and measured lead coordinates ranged from 1.4 to $2 \mathrm{~mm}$ in each axis. These deviations between intended and measured targets are greater than the shift in targeting made because of the MER. Assuming accurate target planning, this may mean that the variation in distance from the intended target that occurs due to the inaccuracy of implantation is more significant in determining the actual anatomical site of the DBS tip than the small trajectory adjustments made on the basis of MER results.

Nearly all patients in our series received both right and left electrode implants during a single surgery. There was no systematic difference in DBS electrode tip location for the implants guided by the StereoPlan compared with the Stealth FrameLink 2.0 programs. The FrameLink program offers the advantage of automatic correction for MR imaging tilt and rotation relative to the AC-PC plane, whereas the StereoPlan requires the surgeon to correct manually for any such tilt or rotation. There was also no systematic difference in DBS electrode tip location for the first compared with the second side implants. This suggests that the results in the first side can be reliably used to determine the target on the second side.

To date, few investigators have attempted to compare the neuroimaging-defined accuracy of STN electrode implantation with clinical outcome. In one recent study ${ }^{24}$ the investigators analyzed the relationship between electrode tip location and the intraoperative stimulation threshold for side effects. A significant correlation was found between stimulation-induced visual, sensory, and presumed corticobulbar (dysarthria and facial contraction) effects and the postoperative MR imaging-determined DBS electrode tip locations. Paresthesias correlated with a medial posterior and superior location, corticobulbar side effects correlated with a more lateral tip location, and visual blurring with a more medial location. The DBS electrode tip locations did not correlate with clinical efficacy.

The stimulation settings used were similar for the large majority of electrodes. This leads us to suggest that the clinical effect of the bulk of the electrodes was similar. Two electrodes in a single patient could be considered as "outliers," because they were the only ones programmed in a bipolar mode with the No. 3 electrode contact as negative. Analysis of the anatomical tip locations does not appear to explain these atypical settings. It is possible that the atypical settings were due to an unusual aspect of this patient's clinical syndrome.

In this study, the placement of STN electrodes, as determined on postoperative MR imaging, did not significantly correlate with clinical outcome. The primary clinical outcome measure was an assessment of the UPDRS motor subscale (Part III), which was determined by an impartial observer who was blinded to stimulator status. When we computed the difference in motor scores, comparing the offmedication, off-stimulation state with the off-medication, on-stimulation state 1 year postoperatively, there was a statistically significant improvement ${ }^{8}$ reflecting effective STN stimulation, but the degree of improvement did not correlate with the variation of the anatomical location of the DBS electrode tip from the intended target.

Other standardized measures of clinical outcome, including the UPDRS activity of daily living subscale (Part II), the 
full UPDRS, ${ }^{7}$ the Hoehn and Yahr stage, ${ }^{9}$ the Schwab and England $^{21}$ activity of daily living capacity, estimates of motor fluctuation severity and duration, dyskinesias, and changes in medication requirements, showed no correlation with the accuracy of STN electrode targeting for the study group. ${ }^{14}$, Although standardized tools are the most valid methods of acquiring outcome data, there is validity in using nonstandardized instruments as well, as has been reported elsewhere. ${ }^{1}$

Our patient self-assessment questionnaire, although not a standardized tool, measured three aspects of outcome (laterality of symptoms, dyskinesia improvement, and symptom severity in the off-medication/stimulation state). These patient-reported outcomes, albeit subjective, seem to us to define discrete groups in each of these three aspects of outcome. The results from our findings indicate that these different outcome groups do not differ based on electrode tip location. Although there may be submillimeter differences in tip location that exist below our ability to detect, we believe that the differences in clinical outcome are more likely due to biological variability. In addition, the results from standardized assessment tools agree with those from our nonstandardized questionnaire; mainly that electrode tip location does not correlate significantly with clinical outcome within the parameters of this study. Therefore, we believe that it was reasonable to include data from our nonstandardized questionnaire in our results, despite the fact that it is not as persuasive or powerful a tool as more standardized measures of clinical outcome.

In a separate analysis of the improvement of UPDRS scores in limbs by stimulation, we found no difference in mean tip location among the electrodes yielding the most compared with the least improvement (unpublished data). These results support the notion that the degree of clinical improvement found in our series was not due to variation of the proximity of the DBS electrode within $3 \mathrm{~mm}$ of the intended target.

Therapy with STN stimulation is anticipated to improve right- and left-sided symptoms to a similar degree. The reversal of symptom asymmetry that occurred in one patient did not seem to be attributable to asymmetry of her DBS electrode tip locations or a discrepancy between her treatment and that of the other patients in this series. Because this patient's symptoms altered from left-dominant to rightdominant, we would expect her right electrode (implanted to affect her left-sided symptoms) to be within two SDs of the mean and her left electrode to be significantly different from the mean, if her clinical outcome was in fact due to electrode placement.

There are several reasons for the apparent lack of correlation between electrode placement accuracy and clinical outcome measures in our series. It is possible that our clinical measurements were not sensitive enough to detect the millimeter-caliber differences in electrode placement that we achieved using these established methods. It is also possible that many other factors, including the patient's clinical characteristics or the duration of follow up, outweighed the effect of electrode placement. Sample size limitations may also have precluded our ability to detect a correlation between electrode tip location and clinical outcome. A more precise quantitative method of outcome assessment, using neurophysiological or kinematic measurements, may be helpful in detecting differences in outcome that are not evi- dent from the clinical assessment methods used in this study. Further analysis of our limb study results (see earlier discussion) may provide such a correlation.

We noted that tremor persisted on one side in one patient, and this was attributed to an electrode that was placed $4 \mathrm{~mm}$ laterally to the intended STN target. When this individual's electrode was moved medially, excellent tremor control was achieved. This result supports the notion that within a radius of 2 to $3 \mathrm{~mm}$ from the center of the STN, stimulating electrodes may provide essentially equivalent clinical benefit, but beyond this distance suboptimal or inadequate clinical benefit results.

This study as well as others does not provide an explanation for the variations between the intended and actual placement of the electrode tip. Given that the electrode target was a known AC-PC coordinate for every patient, why was there a variation, even in millimeters, between the intended and actual locations of the implanted electrodes? The answer may relate to the current limitations of implantation technology, including mechanical inaccuracies of the stereotactic apparatus, discrepancies between atlas and actual anatomical landmarks, inaccuracies of mapping software, minute degrees of intraoperative shifting of the brain within the skull, artifacts in the postimplantation MR imaging studies obtained for electrode tip determination, and deviations in electrode placement, which is performed without physiological guidance for technical reasons. Our results, however, do not indicate that any of these limitations (except, possibly, for brain shift) contributed to significant or systematic errors. Furthermore, it has been noted that not all symptoms of PD respond equally to DBS. It is possible that variations in targeting and electrode placement may have more impact on the control of some PD symptoms than others. Some inaccuracies in targeting, such as those occurring along the $\mathrm{z}$ axis, may have little impact on clinical outcome because the selection of electrode contacts may compensate for locations that are too ventral or dorsal.

Although this study was based on a relatively limited number of cases, we detected no correlation between the clinical outcome and the anatomical location of DBS electrodes, which clustered within a 6-mm-diameter tissue sphere. If image-based targeting alone can provide this degree of accuracy, it remains to be established whether electrophysiological recording, currently regarded as the state of the art, provides an improvement in outcome. Alternatively, minimal use of MER (for example, to confirm electrode placement within some part of the STN) may provide the same efficacy as that achieved by detailed microelectrode mapping to attempt precise placement in the center of the STN. Prospective studies comparing different methods for guiding DBS placement, combined with quantitative postoperative assessments of clinical improvement and DBS electrode location on MR imaging, may be needed to evaluate these issues. To be definitive, such studies might require a larger sample size than our 26 patients (who received 52 STN DBS electrode implants) to offset the possibility that a clinically significant variation of STN shape and location may occur in a small percentage of patients.

\section{Acknowledgments}

We thank Samuel P. Burns of the Columbia Graduate School of Applied Physics and Applied Mathematics for his selfless aid in 


\section{Stimulation of the STN: electrode location and clinical outcome}

\section{APPENDIX}

Novel self-assessment questionnaire administered to patients by mail and telephone interview

Questionnaire

1. What Parkinson's medications are you presently taking?

1a. What are the dosages and time of day that you take them?

2. Did you have significant dyskinesias before the operation?

2a. How are they now?

3. Was your Parkinson's worse on one side (right or left) before the surgery?

3a. Was your Parkinson's worse on one side (right or left) after the surgery?

4. Before the surgery, with a typical dose of Sinemet for how long did you have good function ("good on")?

4a. After the surgery, with a typical dose of Sinemet for how long do you have good function ("good on")?

5. Before the surgery did you have severe "off" times?

5a. If yes, how many times per day and how long did they last?

5 b. Do you have severe "off" times now?

$5 \mathrm{c}$. If yes, how many times per day and how long do they last?

6. Are the "off" times less severe after the surgery?

preparing the necessary computer graphics for this paper. We also thank Brian Kim for his help in meticulously acquiring and compiling the data on STN height and patients' history of surgery for PD that were used in this paper.

\section{Disclaimer}

Except for the National Institutes of Health grant to Dr. McClelland, no author received any financial support in conjunction with this paper.

\section{References}

1. Andrewes DG, Kaye A, Aitken S, et al: The ESDQ: a new method of assessing emotional and social dysfunction in patients following brain surgery. J Clin Exp Neuropsychol 25:173-189, 2003

2. Ashby P, Kim YJ, Kumar R, et al: Neurophysiological effects of stimulation through electrodes in the human subthalamic nucleus. Brain 122:1919-1931, 1999

3. Benabid AL, Pollak P, Gross C, et al: Acute and long-term effects of subthalamic nucleus stimulation in Parkinson's disease. Stereotact Funct Neurosurg 62:76-84, 1994

4. Benazzouz A, Breit S, Koudsie A, et al: Intraoperative microrecordings of the subthalamic nucleus in Parkinson's disease. Mov Disord 17 (Suppl 3):S145-S149, 2002

5. Cotzias GC, van Woert MH, Schiffer LM: Aromatic amino acids and modification of parkinsonism. N Engl J Med 276:374-379, 1967

6. The Deep-Brain Stimulation for Parkinson's Disease Study Group: Deep-brain stimulation of the subthalamic nucleus or the pars interna of the globus pallidus in Parkinson's disease. N Engl J Med 345:956-963, 2001

7. Fahn S, Elton RL: Unified Parkinson's Disease Rating Scale, in Fahn S, Marsden CD, Goldstein M (eds): Recent Developments in Parkinson's Disease. Florham Park, NJ: Macmillan Healthcare Information, 1987, Vol 2, pp 153-163

8. Ford B, Winfield L, Pullman SL, et al: Subthalamic nucleus stimulation in advanced Parkinson's disease: blinded assessments at one year follow up. J Neurol Neurosurg Psychiatry 75: 1255-1259, 2004

9. Hoehn MM, Yahr MD: Parkinsonism: onset, progression, and mortality. Neurology 17:427-442, 1967

10. Hornykiewicz O, Kish SJ: Biochemical pathophysiology of Parkinson's disease. Adv Neurol 45:19-34, 1987

11. Just H, Ostergaard K: Health-related quality of life in patients with advanced Parkinson's disease treated with deep brain stimulation of the subthalamic nuclei. Mov Disord 17:539-545, 2002
12. Krack P, Pollak P, Limousin P, et al: Subthalamic nucleus or internal pallidal stimulation in young onset Parkinson's disease. Brain 121:451-457, 1998

13. Kumar R, Lozano AM, Kim YJ, et al: Double-blind evaluation of subthalamic nucleus deep brain stimulation in advanced Parkinson's disease. Neurology 51:850-855, 1998

14. Langston JW, Widner H, Goetz CG, et al: Core assessment program for intracerebral transplantations (CAPIT). Mov Disord 7:2-13, 1992

15. Limousin P, Krack P, Pollak P, et al: Electrical stimulation of the subthalamic nucleus in advanced Parkinson's disease. N Engl J Med 339:1105-1111, 1998

16. Limousin P, Pollak P, Benazzouz A, et al: Effect of parkinsonian signs and symptoms of bilateral subthalamic nucleus stimulation. Lancet 345:91-95, 1995

17. Marsden CD, Parkes JD: "On-off" effects in patients with Parkinson's disease on chronic levodopa therapy. Lancet 1:292-296, 1976

18. Pinter MM, Alesch F, Murg M, et al: Deep brain stimulation of the subthalamic nucleus for control of extrapyramidal symptoms in advanced idiopathic Parkinson's disease: one year follow-up. J Neural Transm 106:693-709, 1999

19. Saint-Cyr JA, Hoque T, Pereira LC, et al: Localization of clinically effective stimulating electrodes in the human subthalamic nucleus on magnetic resonance imaging. J Neurosurg 97:1152-1166, 2002

20. Schaltenbrand G, Bailey P: Introduction to Stereotaxis With an Atlas of the Human Brain. Stuttgart: Thieme, 1959

21. Schwab RS, England AC: Projection technique for evaluating surgery in Parkinson's disease, in Gillingham FJ, Donaldson MC (eds): Third Symposium on Parkinson's Disease. Edinburgh: E\&S Livingstone, 1969, pp 152-157

22. Simuni T, Jaggi JL, Mulholland H, et al: Bilateral stimulation of the subthalamic nucleus in patients with Parkinson's disease: a study of efficacy and safety. J Neurosurg 96:666-672, 2002

23. Starr PA, Christine CW, Theodosopoulos PV, et al: Implantation of deep brain stimulators into the subthalamic nucleus: technical approach and magnetic resonance imaging-verified lead locations. J Neurosurg 97:370-387, 2002

24. Starr PA, Vitek JL, Bakay RA: Ablative surgery and deep brain stimulation for Parkinson's disease. Neurosurgery 43:989-1015, 1998

25. Starr PA, Vitek JL, Bakay RA: Deep brain stimulation for movement disorders. Neurosurg Clin N Am 9:381-402, 1998

26. Sumanaweera TS, Adler JR Jr, Napel S, et al: Characterization of spatial distortion in magnetic resonance imaging and its implications for stereotactic surgery. Neurosurgery 35:696-704, 1994

27. Vesper J, Klostermann F, Stockhammer F, et al: Results of chronic subthalamic nucleus stimulation for Parkinson's disease: a 1-year follow-up study. Surg Neurol 57:306-313, 2002

28. Voges J, Volkmann J, Allert N, et al: Bilateral high-frequency stimulation in the subthalamic nucleus for the treatment of Parkinson disease: correlation of therapeutic effect with anatomical electrode position. J Neurosurg 96:269-279, 2002

29. Zesiewicz TA, Hauser RA: Neurosurgery for Parkinson's disease. Semin Neurol 21:91-101, 2001

30. Zonenshayn M, Rezai AR, Mogilner AY, et al: Comparison of anatomic and neurophysiological methods for subthalamic nucleus targeting. Neurosurgery 47:282-294, 2000

Manuscript received June 15, 2005.

Accepted in final form September 21, 2005.

This work was partially supported by National Institutes of Health Grant No. T35 HL07616 to Dr. McClelland.

Address reprint requests to: Robert R. Goodman, M.D., Ph.D., Department of Neurological Surgery, Neurological Institute of New York, 710 West 168th Street, Box 99, New York, New York 10032. email: rrg2@columbia.edu. 